\title{
Kif5b Modulates Processing and Secretion of von Willebrand Factor in Endothelial Cells and Mice
}

\author{
Yu-Si Luo ${ }^{1,2,3}$, Yue Zhuo ${ }^{4,5,6 *}$, Ke Zhang ${ }^{7}$, Xingde Liu ${ }^{8}$, Erhard Hofer ${ }^{2}$ and Jian-Dong Huang ${ }^{1,6,9}$ \\ ${ }^{1}$ School of Biomedical Sciences, The University of Hong Kong, Hong Kong SAR, P.R China \\ ${ }^{2}$ Department of Vascular Biology and Thrombosis Research, Medical University of Vienna, Vienna, Austria \\ IIntensive Care Unit, Department of Emergency, Guizhou Medical University Affiliated Hospital, Guiyang, P. R. China \\ ${ }^{4}$ Laboratory of Immune Development and Immune Therapy, Department of Obstetric \& Gynecologic, West China Second University Hospital, Sichuan University, \\ Chengdu, P. R. China \\ ${ }^{5}$ Key Laboratory of Birth Defects and Related Diseases of Women and Children (Sichuan University), Ministry of Education, Chengdu, P. R. China \\ ${ }^{6}$ Institute of Biomedicine and Biotechnology, Shenzhen Institutes of Advanced Technology, Chinese Academy of Sciences, Shenzhen, P. R. China \\ ${ }^{7}$ Key and Characteristic Laboratory of Modern Pathogen Biology, Guizhou Medical University, Department of Education of Guizhou Province \\ ${ }^{8}$ Department of Cardiology, Guizhou Medical University Affiliated Hospital, Guiyang, P. R. China \\ ${ }^{9} \mathrm{HKU}-\mathrm{Shenzhen}$ Institute of Research and Innovation, Shenzhen, P. R. China
}

\begin{abstract}
Von Willebrand factor (VWF) secreted by Weibel-Palade bodies within endothelial cells is critical to maintain normal platelet adhesion during vascular arrest. Because the transportation of VWF is microtubule-dependent partly via kinesin activity, suppression of kinesin could affect intracellular trafficking of VWF. In this study, we investigated the role of Kif5b, the key member of the kinesin superfamily, in the processing and secretion of VWF. A hypothetical interaction between VWF and Kif5b was confirmed and the tail domain of Kif5b was identified as VWF binding region. Knocking-down Kif5b in human umbilical vein endothelial cells led to significantly increased non-stimulated VWF secretion, considerably higher ratio of pro-VWF to mature VWF, and shorter VWF length. Consistent to the in vitro assay, Kif5b knockdown mice demonstrated dramatically increased VWF secretion after epinephrine stimulation. Significantly prolonged bleeding time was observed in these Kif5b knockdown mice as well, which was further elucidated by the decreased basal/regulated VWF secretion in Kif5b knockdown mice comparing to their wild-type littermates. Taken together, these findings suggest that Kif5b modulates processing and secretion of VWF in vitro and in vivo.
\end{abstract}

Keywords: Von Willebrand factor; Human umbilical vein endothelial cells; Kif5b; Kif5b knockdown mice

\begin{abstract}
Abbreviations: BSA: Bovine Serum Albumin; Cy3: Indocarbocianine; DAB: 3,3'-Diaminobenzidine; DAPI: 4',6-Diamidino-2-phenylindole; EDTA: Ethylene Diamine Tetraacetic Acid; EGTA: Ethylene Glycol-bis ( $\beta$-aminoethyl ether) -N, N, N', N'-tetraacetic Acid; ELISA: Enzyme-linked Immunosorbent Assay; EP: Epinephrine; GST: Glutathione S-transferase; HBS: HEPES Buffered Saline; HEK293T: Human Embryonic kidney 293T; HEPES: N-2-hydroxyethylpiperazine-N'-2-ethanesulfonic Acid; HRP: Horseradish Peroxidase; HUVECs: Human Umbilical Vein Endothelial Cells; IPTG: Isopropyl $\beta$-D-1-thiogalactopyranoside; MDCK: Madin-Darby Canine Kidney; NP-40: Nonidet P-40; O/N: Over Night; PBS: Phosphate Buffered Saline; PCR: Polymerase Chain Reaction; SDS-PAGE: Sodium Didedecyl Sulphate-Polyacrylamide Gel Electrophoresis; shRNA: Small Hairpin RNA; TGN: Trans-Golgi Network; UK: United Kingdom; USA: United States of America; VWF: Von Willebrand factor; WPBs: Weibel-Palade Bodies.
\end{abstract}

\section{Introduction}

Physiological balance of the hemostatic system is maintained by the cooperation of procoagulant, anticoagulant, and fibrinolytic factors. Von Willebrand factor (VWF) is one of the key members of the procoagulant system. It is a large, highly adhesive, and multimeric glycoprotein that originates from a $350 \mathrm{kDa}$ monomer called pro-VWF [1]. The pro-VWF monomers are transported to the endoplasmic reticulum, where they dimerize through the formation of disulfide bonds. The dimers continue to polymerize in the Golgi complex, and then further multimerize after endoprotease furin cleavage in the trans-Golgi network (TGN) and post-Golgi compartment to produce mature-VWF [2]. VWF is mainly produced in endothelial cells and stored in specialized intracellular granules called Weibel-Palade bodies (WPBs) $[3,4]$. Besides endothelial cells, approximately $15 \%$ of VWF is synthesized and stored in platelets and megakaryocytes [5]. Previous reports have demonstrated that VWF could be secreted into the plasma via regulated, constitutive, and basal pathways. Both of regulated and basal secretions occur from WPBs and produce highly VWF multimers. However, the constitutive secretion has not been sorted into WPBs whereas high VWF multimerization cannot be undertaken [1]. Among these pathways, both basal and regulated secretion are primarily targeted to the apical side of endothelial cells, whereas constitutive secretion is solely towards to the basolateral side [6]. After secretion into the plasma, the ultra-large VWF multimer will be cleaved into normal molecular weight range VWF by the metalloprotease ADAMTS13 to avoid excessive platelet aggregation $[7,8]$. A number of reports have shown that impaired processing, maturation, storage, and secretion of VWF can cause various diseases, including von Willebrand disease, thrombotic thrombocytopenic purpura, myocardial infarction, and stroke [9-11].

*Corresponding author: Dr. Yue Zhuo, Laboratory of Immune Development and Immune Therapy, Department of Obstetric \& Gynecologic, West China Second University Hospital, Sichuan University. Mailing address: No. 20, Section 3, Renmin South Road, Chengdu, Sichuan, People's Republic of China, 610041, Tel: +8618782921595, E-mail: zhuoy2000@foxmail.com

Received September 21, 2018; Accepted November 05, 2018; Published November 12, 2018

Citation: Yu-Si Luo, Yue Zhuo, Ke Zhang, Xingde Liu, Erhard Hofer, et al. (2018) Kif5b Modulates Processing and Secretion of von Willebrand Factor in Endothelial Cells and Mice. Cardiovasc Pharm Open Access 7: 249. doi: 10.4172/23296607.1000249

Copyright: ( 2018 Luo YS, et al. This is an open-access article distributed under the terms of the Creative Commons Attribution License, which permits unrestricted use, distribution, and reproduction in any medium, provided the original author and source are credited. 
Although the process of VWF exocytosis is not completely clear, previous research has indicated that VWF secretion is dependent on kinesins [12]. Furthermore, Manneville et al. found that non-specific inhibition of all kinesins could sharply decrease VWF transportation velocity to the plasma membrane [13]. However, how kinesins modulate VWF secretion keeps poorly understood [1].

Kinesin-1, also known as conventional kinesin, consists of two heavy chains and two light chains [14]. Kinesin-1 uses microtubules as a "railway" for transportation, and is classified as a microtubule-plusend motor protein [15]. Three different kinesin-1 heavy chains have been reported, Kif5a, Kif5b, and Kif5c, in which Kif5b is ubiquitously expressed in mammals [16]. The expression pattern of Kif5b suggests that it is critical for intracellular trafficking $[17,18]$. The role of Kif5b in neuronal activity has been widely studied. It has been reported that Kif5b associates with various proteins involved in neuronal development, morphology, plasticity, and survival [17,19-21]. In contrast, there is relatively scarce information about Kif5b in non-neuronal tissues $[22,23]$. Our group had reported that specifically inactivating Kif5b in pancreatic cells led to deficient insulin secretion in vivo [24]. The work of another group indicated that Kif5b affected the assembly of myofibril components and their linkage to the myotendinous junctions [25].

Notably, some studies reported that the disruption of microtubules blocked VWF transportation in endothelial cells [26,27]. Furthermore, non-specific inhibition of kinesins caused a decrease in the frequency of VWF long-range movements along microtubules [13]. However, to our knowledge, few publications addressed the relationship between Kif5b and VWF.

In this study, we generally explored the roles of Kif5b in the processing and secretion of VWF in vitro via Kif5b knockdown human umbilical vein endothelial cells (HUVECs) and in vivo via Kif5b knockdown mice (Kif5b $\mathrm{b}^{+/}$mice).

\section{Materials and Methods}

\begin{abstract}
Animals
$\mathrm{Kif}_{5} \mathrm{~b}^{+/-}$mice were kindly provided by Dr. ZG Duan and fed with pelleted diet and water ad lib. Generation of $\mathrm{Kif}_{5} \mathrm{~b}^{+/-}$mice was carried out by gene targeting and genotyping $[24,28]$. To generate Kif $5 \mathrm{~b}^{+/-}$mice, wild-type mice (termed as $\mathrm{Kif}_{5} \mathrm{~b}^{+/+}$mice) were crossed with $\mathrm{Kif5}^{\mathrm{b}} \mathrm{H}^{+-}$ mice. Genomic DNA samples were extracted from mouse ear as PCR templates for genotyping [24]. All animal experiments were performed under the guidelines of the Committee on the Use of Live Animals in Teaching and Research (CULATR \#2540-11) at The University of Hong Kong.
\end{abstract}

\section{Cells}

HUVECs and related culture medium were purchased from Thermal Fisher Scientific. HUVECs were grown in Medium200 (M200500, Thermal Fisher Scientific, USA) according to the attached protocol of the Angiogenesis Starter Kit (A14609-01, Thermal Fisher Scientific, USA). HEK293T cells were cultured as previously described [29]. All experiments using HUVECs were performed on cells from less than five passages. All cells were cultured at $37^{\circ} \mathrm{C}$ in the presence of $5 \% \mathrm{CO}_{2}$.

\section{Glutathione S-Transferase (GST) pull-down assay}

Four Kif5b fragments (Kif5b-a, Kif5b-b, Kif5b-c, and Kif5b-d) fused to GST were generated as previously described [25]. Kif5b-a, Kif5b-b, Kif5b-c, and Kif5b-d corresponding to the 1-413, 414-678, 679-849, and 850-963 amino acid fragments, representing the motor domain, the stalk domain, the kinesin light chain binding domain, and the C-terminus of the tail domain of Kif5b, respectively (Figure 1A). The four GST-fusion fragments were expressed in Escherichia coli BL21 host cells. Protein expression was induced by introducing $0.5 \mathrm{mM}$ IPTG when the optical density of BL21 host cells at $600 \mathrm{~nm}$ reached 0.6-0.7. Cells were harvested by centrifugation, and the cell pellet was resuspended and washed in PBS. The pellet was resuspended in HBS and sonicated on ice to release soluble proteins. The bacterial lysate was centrifuged at $4^{\circ} \mathrm{C}$ to remove cell debris, and the supernatant was incubated with glutathione-Sepharose 4B beads (17075605, Amersham Pharmacia Biotech, UK) at $4^{\circ} \mathrm{C}$ for $1 \mathrm{~h}$. Next, HUVECs were washed with PBS, and then scratched and lysed in HBS on ice. The lysate of HUVECs was centrifuged at $4^{\circ} \mathrm{C}$ to remove debris, and then incubated with GST-protein-bound beads at $4^{\circ} \mathrm{C} \mathrm{O} / \mathrm{N}$. The beads were then removed by centrifugation at $4^{\circ} \mathrm{C}$. The supernatant was subjected to SDS-PAGE and immmunoblotting using 1:10,000 diluted primary antibody against VWF (A0082, DAKO, Denmark) and then 1:10,000 diluted secondary antibody HRP-conjugated goat anti-rabbit (65-6120, Thermo Fisher Scientific, USA). Simultaneously, the gel was stained by Coomassie Blue reagent for 2 to $3 \mathrm{~h}$, and then destained by destaining buffer.

\section{Plasmids and lentiviral vectors}

In order to construct the Kif5b knockdown HUVECs for investigating the potential relationship between Kif5b and VWF, firstly we designed the shRNAs for decreasing the expression of Kif5b and finally we were gifted the shRNA plasmid for Kif5b knockdown and the scrambled shRNA plasmid for control from Dr. MF Liu. The plasmid for Kif5b shRNA (pLL3.7-shRNA Kif5b) was confirmed by sequencing with the sense primer 5'-CAGCACAAAAGGAAACTCACC-3' and antisense primer 5'-GGCGGTAATACGGTTATCCAC-3'. Kif5b

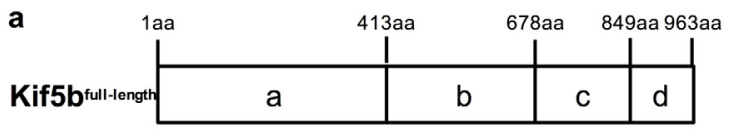

b

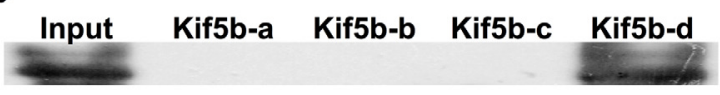

Kif5b-a Kif5b-b Kif5b-c Kif5b-d

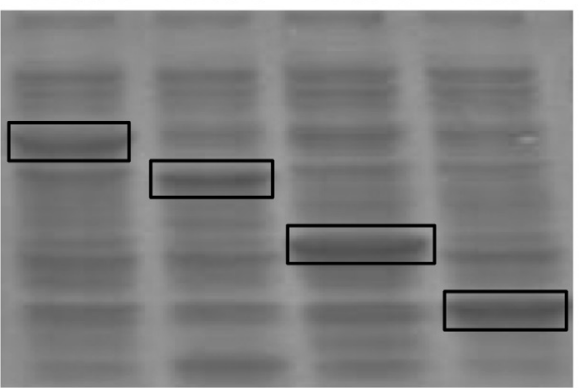

Figure 1: Kif5b interacted with VWF through its tail domain. (A) Schematic figure of Kif5b truncations. (B) Kif5b C-terminus tail domain interacted with VWF. GST-tagged recombinant Kif5b-d fragment was able to pull down VWF from HUVECs lysates. (C) The expressions of 4 GST-fusion Kif5b fragments (the expressed Kif5b-a, Kif5b-b, Kif5b-c, and Kif5b-d fragments were indicated by black frames). 
shRNA lentiviruses were generated from pLL3.7-shRNA Kif5b using standard methods in subconfluent HEK293T cells [30]. Briefly, the HEK293T cells were co-transfected with the pLL3.7-shRNA Kif5b plasmid and helper plasmids (pGag/Pol, pVSVG, and pREV) using Lipofectamine 3000 (Thermal Fisher Scientific, USA). The supernatant samples containing virus were harvested after $48 \mathrm{~h}$. HUVECs were infected with the virus supernatant by incubation for $24 \mathrm{~h}$, and then cultured in fresh growth medium. After 5 days, cells surviving from 2 $\mu \mathrm{g} / \mathrm{mL}$ puromycin (Sigma-Aldrich, USA), termed as Kif5b-knockdown HUVECs, were selected and used in the following assays. The control shRNA lentivirus was packaged, collected, and inoculated into the HUVECs to generate the control HUVECs as same as the generation of Kif5b-knockdown HUVECs.

\section{ELISA analysis of VWF secretion}

To evaluate VWF levels in supernatant samples from cell culture contents of Kif5b-knockdown HUVECs and the control HUVECs, we performed ELISA assay stepwise. The 24-well tissue culture plates were used to culture the cells until they reached $80 \%$ confluence as $3 \times 10^{5} /$ well. To evaluate VWF secretion, cells were starved in Medium 200 for $1 \mathrm{~h}$. Cells were washed with PBS and cultured with fresh Medium 200 containing $100 \mu \mathrm{M}$ histamine (H7250, Sigma-Aldrich, USA) at $37^{\circ} \mathrm{C}$ for $30 \mathrm{~min}$. After removing cell debris by centrifugation for $5 \mathrm{~min}$, the supernatant was collected and stored at $-20^{\circ} \mathrm{C}$.

To evaluate VWF levels in plasma samples of $\mathrm{Kif} \mathrm{b}^{+/-}$mice and $\mathrm{Kif}_{5} \mathrm{~b}^{+/+}$mice, epinephrine (EP) (E4642, Sigma, USA) was subcutaneously injected into $6 \mathrm{Kif} \mathrm{b}^{+/-}$mice and $6 \mathrm{Kif} \mathrm{b}^{+/+}$mice at a dose of $1 \mathrm{mg} / \mathrm{kg}$ body weight. Whole blood samples were taken via submandibular bleeding and drawn into $3.2 \%$ sodium citrate before and $30 \mathrm{~min}$ after EP injection as described elsewhere [31]. Platelet-poor plasma was then obtained by centrifugation at $1,500 \mathrm{~g}$ for $15 \mathrm{~min}$. A standard curve was plotted based on the pooled plasma from five male $\mathrm{Kif} \mathrm{b}^{+/+}$mice, and the concentration of VWF was assumed as $10 \mu \mathrm{g} /$ $\mathrm{mL}[32,33]$.

Both cell supernatant and mice plasma VWF levels were measured by ELISA using the rabbit polyclonal antibody against VWF $(10 \mu \mathrm{g} / \mathrm{mL})$ (A0082, DAKO, Denmark) and the rabbit polyclonal anti-human VWF antibody conjugated with HRP (1:8,000) (P0226, DAKO, Denmark). The color was developed using tetramethylbenzidine solution (Sigma, USA) and the optical density at $450 \mathrm{~nm}$ was measured using an ELISA reader (Sunrise, Tecan Trading AG, Switzerland). The ELISA results were evaluated as units per milligram of total cell protein.

\section{Protein expression analysis}

To confirm the knockdown of Kif5b and demonstrate consequent expression of VWF, the extraction, electrophoresis, and Western blot assay were performed with the protein samples from Kif5bknockdown HUVECs, control HUVECs, Kif5 $\mathrm{b}^{+/-}$mice, and Kif5 $\mathrm{b}^{+/+}$ mice, respectively. Briefly, Kif5b-knockdown HUVECs and the control HUVECs, respectively, were scratched from the plate and lysed using ice-cold HBS buffer (20 mM HEPES pH 7.2, $150 \mathrm{mM} \mathrm{NaCl}, 2$ mM EDTA, 2 mM EGTA, and 0.5\% NP-40). Mouse aorta tissue samples of $\mathrm{Kif}_{5} \mathrm{~b}^{+/}$and $\mathrm{Kif}_{5} \mathrm{~b}^{+/+}$mice were homogenized and lysed using HBS buffer on ice for $30 \mathrm{~min}$. The supernatant was collected by centrifugation at $4^{\circ} \mathrm{C}$ and stored at $-20^{\circ} \mathrm{C}$. Total protein concentrations of cells and tissue lysates were determined by BCA protein assay (Thermo Fisher Scientific, USA). Protein samples with equal amounts of total protein were loaded onto SDS-PAGE gels for electrophoresis and subsequent Western blot analysis. Protein expression levels were determined by Western blotting with a primary rabbit anti-Kif5b monoclonal antibody $(1: 2,000)$ [24] and a primary rabbit anti-VWF polyclonal antibody (1:10,000) (A0082, Dako, Denmark). The 1:5,000 diluted anti- $\alpha$-tubulin antibody (T6199, Sigma-Aldrich, USA) was used to detect the expression level of tubulin acted as a loading control. The HRP-conjugated goat anti-rabbit antibody (65-6120, Thermo Fisher Scientific, USA) was used as the secondary antibody for Kif5b and VWF. The HRP-conjugated goat anti-mouse antibody (62-6520, Thermo Fisher Scientific, USA) was used as the secondary antibody for tubulin. The immunoreactive bands were developed and visualized by Pierce Enhanced Chemiluminescence Kit (Thermo Fisher Scientific, USA) and CL-XPosure Film (Thermo Fisher Scientific, USA). The expression levels of Kif5b and VWF were determined using the standard intensity function in the open source Fiji software (ImageJ V1.49, http://fiji.sc/Fiji).

To investigate distribution of constitutive, basal, and regulated secretion of VWF from Kif $5 b^{+/-}$and Kif5 $b^{+/+}$mice, the VWF multimer analysis was carried out as previously described [34]. Briefly, whole blood samples were taken via submandibular bleeding from Kif5 $\mathrm{b}^{+/-}$ and $\mathrm{Kif} \mathrm{b}^{+/+}$mice and immediately drawn into $3.2 \%$ sodium citrate as described elsewhere [31]. Platelet-poor plasma was then obtained by centrifugation at $1,500 \mathrm{~g}$ for $15 \mathrm{~min}$ at room temperature. Total protein concentrations of samples were determined by BCA protein assay. Protein samples with equal amounts of total protein were loaded onto gels for reducing gel electrophoresis and subsequent Western blot analysis. For reducing gel electrophoresis, a Hoefer SE600 ruby standard dual-cooled gel electrophoresis unit (GE Healthcare Life Sciences, Austria) was used. The stacking gel was set with final concentration of $0.8 \% \mathrm{wt} / \mathrm{vol}$ Seakem high gelling temperature agarose (50040, Lonza Ltd, USA), $70 \mathrm{mM}$ Tris base (pH 6.8), 4 mM EDTA disodium salt, and $0.4 \% \mathrm{wt} / \mathrm{vol}$ SDS. The separating gel was set with final concentration of $2 \% \mathrm{wt} / \mathrm{vol}$ Seakem high gelling temperature agarose, 0.1 M Tris base, $0.1 \mathrm{M}$ glycine ( $\mathrm{pH} 8.8$ ), and $0.4 \% \mathrm{wt} / \mathrm{vol}$ SDS. The running buffer (50 $\mathrm{mM}$ Tris base, $0.384 \mathrm{M}$ glycine, and $0.1 \%$ SDS) was included in lower buffer chamber of the electrophoresis unit, and the upper buffer chamber contained $600 \mathrm{~mL}$ of running buffer. After $10 \mathrm{uL}$ sample loading, the gels were run at $10 \mathrm{~mA}$ constant current for 30 min then $13 \mathrm{~mA}$ constant current for $4 \mathrm{~h}$ under $9^{\circ} \mathrm{C}$ with a circulating water bath. The $0.45-\mu \mathrm{m}$ Hybond-LFP low-fluorescent PVDF membranes were presoaked in methanol for $1 \mathrm{~min}$ and then stored in transfer buffer (2.5 mM Tris ( $\mathrm{pH} \mathrm{8.8),} 19.2 \mathrm{mM}$ glycine, $20 \%$ methanol, and $0.01 \%$ SDS) until use. The proteins were transferred to the methanol activated PVDF membranes with the transfer buffer under $12 \mathrm{~V}$ constant voltage for $3 \mathrm{~h}$ and stepwise labeled with a primary rabbit anti-VWF polyclonal antibody (1:20,000) (A0082, Dako, Denmark) $\mathrm{O} / \mathrm{N}$ followed by a incubation of 1:8,000 diluted HRP-conjugated goat anti-rabbit antibody (65-6120, Thermo Fisher Scientific, USA) for $4 \mathrm{~h}$. The signals were developed with Luminata Crescendo Western HRP Substrate (Millipore Ltd, USA), exposed on KODAK BioMax Scientific Imaging Film and visualized with the KODAK medical X-Ray Processor (Eastman Kodak, USA).

\section{Immunohistochemical and immunocytochemical analysis of VWF in aorta tissue and HUVECs}

To detect the expression of VWF in situ in aorta tissue, histological examinations of mouse aorta tissues of $\mathrm{Kif} \mathrm{b}^{+/-}$and $\mathrm{Kif}^{+} \mathrm{b}^{+/+}$mice were carried out according to a previous report [35] with some modifications. Briefly, polyclonal rabbit antibody against VWF (1:400) (A0082, Dako, Denmark) was introduced as the primary antibody. The VWF was visualized with liquid 3,3'-diaminobenzidine (DAB) and substrate-chromogen solution from the EnVision+System/HRP 
(DAB) Kit (K400611, Dako, Denmark), and counter-stained with hematoxylin. The slides were photographed using a Nikon MicrophotoFX microscope attached to a Nikon digital camera.

To precisely present the location and morphology of VWF in cell, immunocytochemical analysis of VWF in Kif5b-knockdown HUVECs and the control HUVECs were performed as previously described [32] with minor modifications. After HUVECs reached 30\% confluence, the cells were fixed with $4 \%$ paraformaldehyde on ice for $15 \mathrm{~min}$. Cells were permeabilized with blocking buffer (5\% Serum, 3\% BSA, $0.25 \%$ TritonX-100) for an additional $10 \mathrm{~min}$, and then incubated with the primary antibody (1:200) (A0082, DAKO, Denmark) in blocking buffer at $4^{\circ} \mathrm{C}$ overnight. On day 2 , the cells were stained with secondary antibodies Alexa Fluor 488 (1:400) (Thermo Fisher Scientific, USA) and Cy3 $(1: 10,000)$ (Jackson Immuno-Research Laboratories, USA), and mounted in SlowFade Gold antifade reagent with DAPI (Thermo Fisher Scientific, USA). Fluorescence was detected and visualized with a Carl Zeiss LSM 700 confocal microscope.

\section{Hematological analysis}

To evaluate bleeding time, $14 \mathrm{Kif}_{5} \mathrm{~b}^{+/-}$mice and $14 \mathrm{Kif}^{\mathrm{b}} \mathrm{b}^{+/+}$mice were anesthetized with ketamine $40 \mu \mathrm{g} / \mathrm{g}$ body weight and xylazine 6 $\mu \mathrm{g} / \mathrm{g}$ body weight via intraperitoneal injection. About $1 \mathrm{~cm}$ length of the distal tail was quickly severed with sharp scissors. The bleeding tail was immersed in PBS at $37^{\circ} \mathrm{C}$ and the time spans between start of bleeding and $30 \mathrm{sec}$ after complete stop of bleeding were recorded as previously reported [31].

\section{Statistical analysis}

Student's $t$-test was utilized for data analysis. Differences were considered significant if $p<0.05$.

\section{Results}

\section{The tail domain of Kif5b directly interacts with VWF in vitro}

To investigate if Kif5b interacts with VWF and to determine which domain interacts with VWF, we examined four GST fusion Kif5b fragments immobilized on Glutathione-Sepharose $4 \mathrm{~B}$ beads in the pull down assay using HUVECs lysates. Only Kif5b-d was able to pull down VWF (Figure 1B), indicating Kif5b interacts with VWF through its C-terminal tail domain. As control, the expression of the four fusion proteins is shown in Figure 1C by Coomassie Blue staining assay.

\section{Knockdown of Kif5b lead to significantly increased VWF} protein secretion in vitro

To address whether Kif5b regulates VWF processing, lentiviral shRNA-mediated knockdown of Kif5b in HUVECs was performed, which led to a remarkable reduction in Kif5b expression (Figure 2A and $\mathrm{B}$ ) and an increase in VWF secretion (Figure 2C). Pro-VWF can be distinguished from mature-VWF based on differences in their electrophoretic mobility [33]. Significant differences in the ratio of proVWF and mature-VWF were observed between the Kif5b-knockdown HUVECs group and the control HUVECs group (Figure $3 \mathrm{~A}$ and $\mathrm{B}$ ). Because pro-VWF precursor undergoes furin-dependent proteolytic cleavage to mature-VWF within the trans-Golgi network before secretion [1], these results indicate that Kif5b is involved in VWF protein processing.

It has been reported that VWF is secreted into the plasma via basal, constitutive, and regulated pathways, and histamine stimulates both central and peripheral VWF granule secretion through increasing intracellular free calcium ion concentration [36]. Significantly increased non-stimulated and induced VWF secretion after $30 \mathrm{~min}$ of histamine stimulation were observed in the Kif5b knockdown HUVECs group compared to the control HUVECs group. This result indicates that knockdown of Kif5b significantly increases VWF secretion in HUVECs (Figure 2C).

\section{Knockdown of Kif5b causes shorter VWF in morphology in vitro}

The classic rod shaped VWFs (indicated by white arrows) in both the Kif5b-knockdown HUVECs and the control group were shown in Figure 4A. These rod shape VWFs, together with other smaller round shaped VWFs, were located around the cell nucleus. Difference in the length of VWFs was observed between the two groups, with $1.3594 \pm$ $0.240 \mu \mathrm{m}$ in Kif5b-knockdown HUVECs while $1.5894 \pm 0.141 \mu \mathrm{m}$ in

a
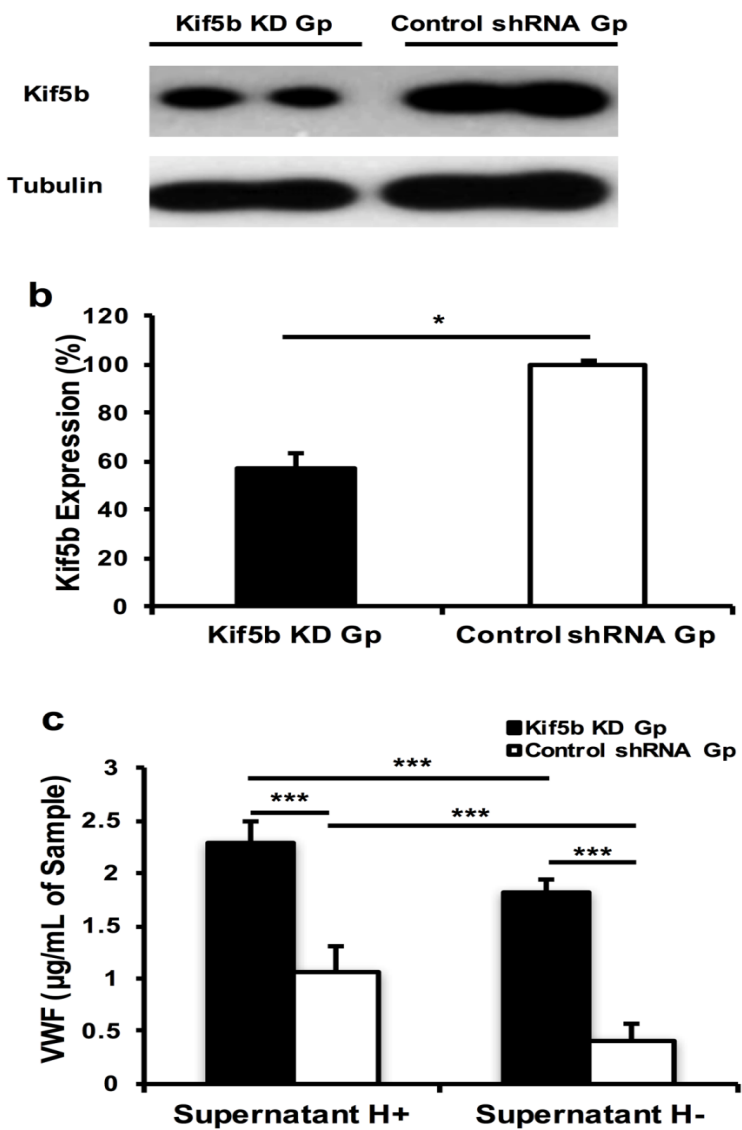

Figure 2: Kif5b was involved in the secretion of VWF in vitro. (A) Western blot analysis indicated Kif5b expression was suppressed in the Kif5bknockdown HUVECs. Tubulin was used as the loading control. Cell population $=3.6 \times 10^{6}$. (B) Quantification of Kif5b expression reduction based on three separate replicates. Each replicate's cell population=1.2 $X 10^{6}$. (C) ELISA results indicated significant changes in VWF secretion in HUVECs 30 min after histamine stimulation. The ELISA results were based on three separate replicates. Each replicate's cell population=1.2 $X 10^{6}$. Kif5b KD Gp: Kif5b-knockdown HUVECs group; Control shRNA Gp: control HUVECs group; Supernatant $\mathrm{H}^{+}$: supernatant with histamine stimulation; Supernatant $\mathrm{H}$ : supernatant without histamine stimulation; Tubulin was used as the loading control; *: $p<0.05$; ${ }^{* *}$ : $p<0.005$. 
a

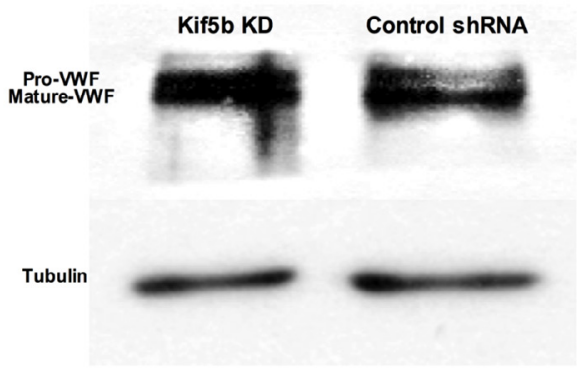

b

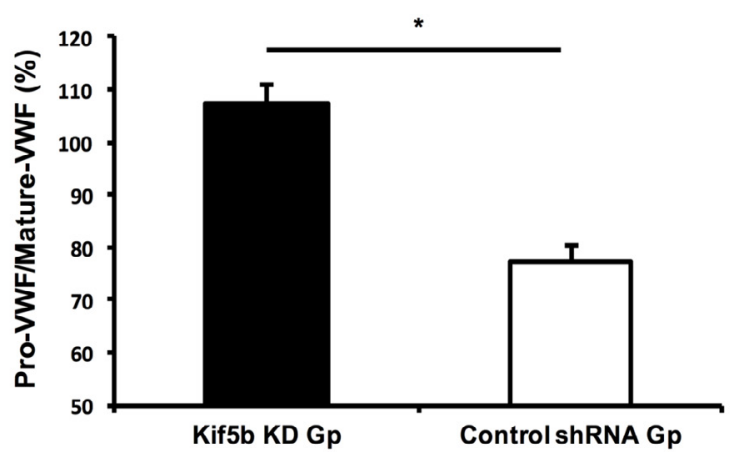

Figure 3: Kif5b was involved in the processing of VWF in vitro. (A) The amount of intracellular pro-VWF and mature-VWF in the Kif5bknockdown HUVECs and the control HUVECs determined by Western blot analyses. This result representative 3 independent assays. Each replicate's cell population $=1.2 \times 10^{6}$. (B) The ratio of pro-VWF/ mature-VWF in the Kif5b-knockdown HUVECs increased significantly compared to the control HUVECs. The ratio was determined by ImageJ (V1.49, http://fiji.sc/Fiji). The results were independently repeated twice. Each repeat's cell population $=1.2 \times 10^{6}$. Kif5b KD Gp: Kif5b-knockdown HUVECs group; Control shRNA Gp: control HUVECs group; *: $p<0.05$.

the control group (Figure 4B). Although the difference in the length of VWF was not statistically significant $(\mathrm{p}>0.05)$, we did observe distinct tabulation of VWF that exhibited in the images. This finding indicates that knockdown of Kif5b causes shorter VWF in morphology in vitro and suggests Kif5b is involved in VWF protein morphology determination to some extend.

\section{Knockdown of Kif5b remarkably promotes VWF release in vivo after epinephrine stimulation}

The transgenic $\mathrm{Kif} \mathrm{b}^{+/-}$mouse line was confirmed by genotyping as previously described (Figure 5A), and Kif5b expression level in $\mathrm{Kif}_{5} \mathrm{~b}^{+/-}$mice was shown to be significantly reduced (Figure 5B). Initial immunohistochemical analysis did not indicate a significant change in VWF expression level or aortal architecture in Kif5 $\mathrm{b}^{+/-}$mice compared to the wild-type littermates (Figure 5C). To determine whether Kif5b knockdown affects VWF secretion, platelet-poor plasma before and $30 \mathrm{~min}$ after EP injection were collected for the evaluation of VWF secretion levels by ELISA. EP stimulation is microtubule-independent and only stimulates peripheral VWF secretion through the elevation of intracellular cyclic adenosine monophosphate (cAMP) level [37]. There were no significant differences in the basal plasma VWF levels between $\mathrm{Kif}_{5} \mathrm{~b}^{+/-}$and Kif5 $\mathrm{b}^{+/+}$mice (Figure 6A). However, after EP stimulation, Kif5 $\mathrm{b}^{+/-}$mice exhibited significantly more VWF secretion than their wild-type littermates (Figure 6A). These results indicate that knockdown of Kif5b remarkably promotes VWF secretion in vivo.
Knockdown of Kif5b significantly delays hemostasis in vivo via less high molecular weight VWF multimer expression

Although the basal secretion of VWF was not significantly affected by Kif5b knockdown, we found that $\mathrm{Kif} \mathrm{b}^{+/-}$mice exhibited remarkably longer bleeding time than $\mathrm{Kif}_{5} \mathrm{~b}^{+/+}$mice (Figure 6B), which indicates that inhibition of Kif5b significantly hampers hemostasis in vivo. Subsequently, the multimer analysis of VWF showed that the Kif5 $\mathrm{b}^{+/}$ mice expressed less basal/regulated source VWF than $\mathrm{Kif}^{\mathrm{b}} \mathrm{b}^{+/+}$mice, as shown in Figure 6C.

\section{Discussion}

Previous reports demonstrated that Kif5b plays important roles in intracellular transportation, cell division, and organelle positioning $[38,39]$. Kinesin-1 binds to its cargo through protein-protein interactions [40], with the C-terminal domain of Kif5b [14,41,42]. Consistent with these reports, our results identify that Kif5b actually interacts with VWF protein through its C-terminus tail domain (within the 850-963 amino acid).

However, it is still unclear to what extent and how kinesins regulate VWF processing, maturation, and secretion. One line of the experiments was directed to investigate this in vivo. The Kif5 $\mathrm{b}^{+/-}$mice used to explore the relationship between Kif5b and VWF in vivo were generated by geno-targeting. Geno-targeting can simulate endogenous

a

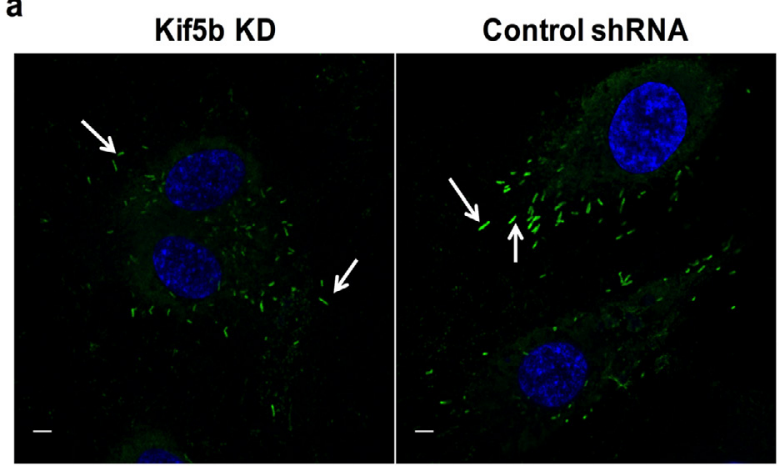

b

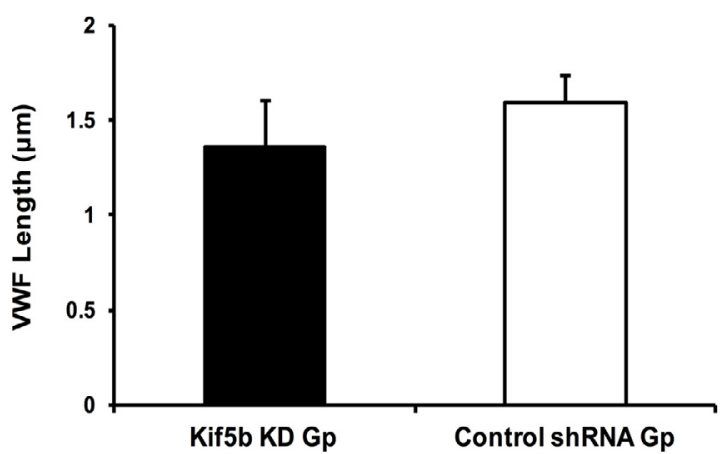

Figure 4: Kif5b knockdown did not significantly change VWF morphology but shorter length of VWF was shown in Kif5b-knockdown HUVECs. (A) Two representative confocal images showed VWF morphology in Kif5bknockdown HUVECs and the control HUVECs. The white arrows indicated VWF. The number of images of each group $=3$. (B) The maximum VWF length in the Kif5b-knockdown HUVECs $(n=112)$ were comparable to the maximum VWF length in the control HUVECs $(n=105)$. Kif5b KD Gp: Kif5b-knockdown HUVECs group; Control shRNA Gp: control HUVECs group; Scale bar $=50 \mu \mathrm{m}$. 


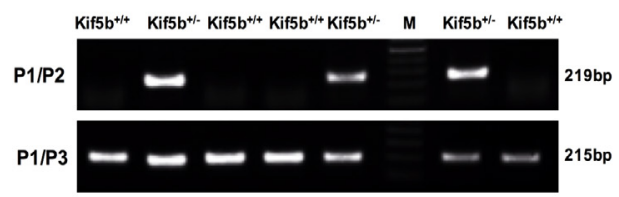

b

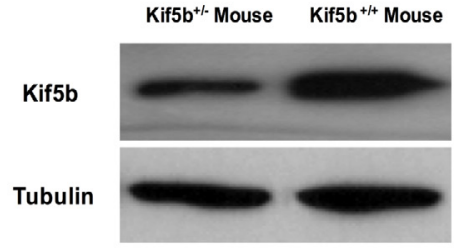

C

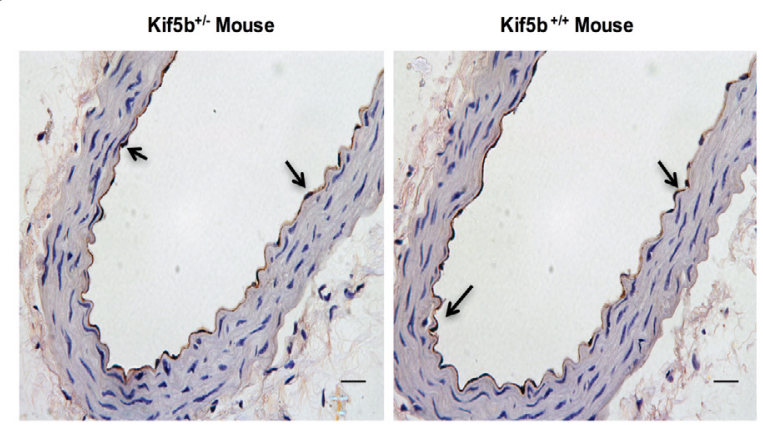

Figure 5: Transgenic Kif5b knockdown mice. (A) PCR results for the genotyping of offspring genomic DNA from mice ear. DNA samples producing 219 bps products with the P1/P2 primer set and 215 bps products with the P1/P3 primer set represented Kif5b knockdown alleles, whereas samples producing only 215 bps products with the P1/P3 primer set represented wide-type alleles. (B) Western blot analysis of aorta tissues indicated reduced Kif5b expression in $\mathrm{Kif5}^{+/-}$mice compared to Kif5 $\mathrm{b}^{+/+}$ mice. Tubulin was used as the loading control. (C) Aorta tissue from Kif5 $\mathrm{b}^{+/-}$ and $\mathrm{Kif} \mathrm{b}^{+/+}$mice showed no significant morphological changes and VWF expression difference. Aorta tissues were immunohistochemically stained for total VWF (brown color indicated by black arrows). Kif5 $\mathrm{b}^{+/+}$mice: wildtype mice; Kif5 ${ }^{+/-}$mice: Kif5b knockdown mice; M: DNA marker; Scale bar $=20 \mu \mathrm{m}$.

disease in vivo better than other methods used such as introducing anti-Kif5b antibodies [43] or overexpression of a dominant-negative Kif5b construct. As mentioned before, under normal physiological conditions, secretion of VWF with appropriate multimer structures, especially the high molecular weight multimers of VWF ranging from 5,500 to $10,000 \mathrm{kDa}$, in sufficient amount is highly responsible for normal hemostasis via platelet adhesion and aggregation upon vascular trauma $[44,45]$. When we investigated $\mathrm{Kif} 5 \mathrm{~b}^{+/-}$mice, it was striking, that these mice showed significantly longer bleeding time than wild-type mice, while only slight differences in non-stimulated VWF secretion was presented. However, the multimer analysis of VWF for $\mathrm{Kif}^{\mathrm{b}} \mathrm{b}^{+-}$mice demonstrated less high molecular weight multimer of VWF expression in VWF multimer pattern than Kif5 $\mathrm{b}^{+/+}$mice. Such data suggested the knockdown of Kif5b could be associated with VWF's processing and secretion, especially in the basal and regulated routes, which results in impaired hemostasis capability of mice. Then $\mathrm{EP}$ was given to the mice to extend the observation for regulated VWF secretion in vivo, which only mediates peripheral VWF secretion by up-regulating cAMP concentration [27]. In this case, the $\mathrm{Kif}_{5} \mathrm{~b}^{+/-}$mice exhibited more EP-stimulated VWF releasing compared with the $\mathrm{Kif} \mathrm{b}^{+/+}$mice. Because VWF is mainly expressed in endothelial cells
[5], we further dissected aorta tissue from Kif5b $\mathrm{b}^{+/-}$and $\mathrm{Kif}_{5} \mathrm{~b}^{+/+}$mice. However, no impaired morphology of aorta was observed, and no VWF expression abnormality was detected.

The second line of experiments was performed using shRNAmediated knockdown of Kif5b in HUVEC. In this case, we found that knockdown of Kif5b in HUVECs significantly increased the amount of secreted VWF in the cell supernatants as well as the ratio of proVWF to mature-VWF in the cell lysates, while no obvious changes in the rod-shaped morphology of VWF [46] was observed. The increased ratio of pro-VWF to mature-VWF in Kif5b knockdown HUVECs suggested that Kif5b participated in the processing and secretion of VWF. Take together the result of VWF multimer analysis of in vivo samples, absence of Kif5b would affect not only the amount of VWF in peripheral circulation system, but the physiological functions of VWF in hemostasis. The normal functions of VWF in hemostasis such as collagen binding and platelet binding were impaired as the multimer size of VWF decreases upon Kif5b knockdown. As reported by Stockschlaeder et al. regulated pathway contributes to almost $80 \%$ of functional mature-VWF. And basal secretion is also responsible for hemostatic activity, while constitutive secretion contributes the least to hemostasis as it possesses low molecular weight VWF multimers compared with high and intermediate molecular weight VWF multimers that present in basal and regulated secretions [47].
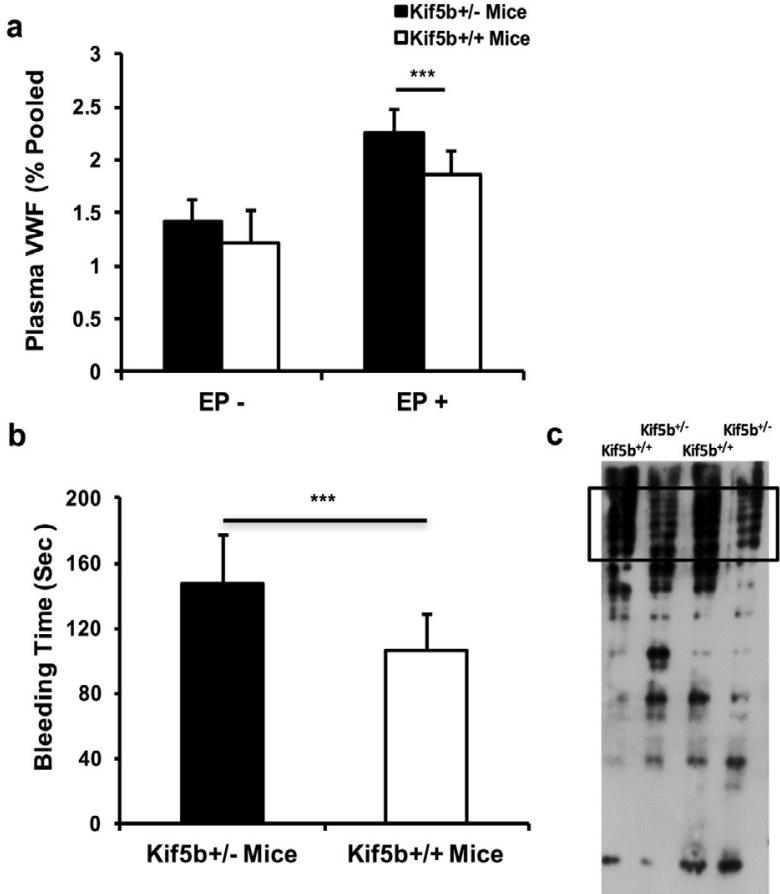

Figure 6: Knockdown of Kif5b altered secretion of VWF in mice. (A) Plasma VWF levels in Kif5 $\mathrm{b}^{+-}$mice $(n=6)$ and $\mathrm{Kif5}^{+/+}$mice $(n=6)$ showed no significant differences before EP injection, but plasma VWF levels in $\mathrm{Kif5}^{+/-}$mice $(\mathrm{n}=6)$ significantly increased after EP injection than Kif5 $\mathrm{b}^{+/+}$ mice $(n=6)$. Optical density values were expressed relative to the VWF level in pooled plasma from $5 \mathrm{Kif5}^{+/+}$mice. (B) Bleeding time in the Kif5 $b^{+/-}$mice $(n=14)$ was significantly longer than in Kif5 $b^{+/+}$mice $(n=14)$. The bleeding assay was conducted twice with 7 mice in each group per assay. (C) VWF multimer assay was carried out with platelet-poor plasma samples from Kif5 $\mathrm{b}^{+/-}$mice $(n=2)$ and $\mathrm{Kif5}^{+/+}$mice $(n=2)$. The black box indicates VWF multimers with high molecular weights. Kif5 $\mathrm{b}^{+/+}$mice: wildtype mice; Kif5b ${ }^{+/-}$mice: Kif5b knockdown mice; EP: epinephrine; $\mathrm{EP}^{+}$. plasma with EP stimulation; EP:: plasma without EP stimulation; Black box: High molecular weight multimer of VWF; ${ }^{* * *}: p<0.005$. 
Nevertheless, the significantly increased VWF secretion upon knockdown of Kif5b expression in the cellular assay seems to be contradictory to the prolonged bleeding time in vivo, and not consistent to the well-known fact that Kif5b is a plus-end motor. The knockdown of Kif5b might also comply with the plus-end motor that should lead to the substantial intracellular drop of VWF. However, the secretion of VWF has a complicated process consisting of basal, constitutive, and regulated pathways [44]. Because of the key and fundermental roles of VWF in normal phsyology, any positive or negative fluctuation of VWF concentration in circulation system will be quickly adjusted. For instance, in our studies, the basal and regulated secretions were blocked by knocking-out Kif5b, then the constitutive pathway might be promoted. However, the pro-VWF and low molecular weight VWF multimers in constitutive pathway play a much less important role in the procoagulant system comparing to basal and regulated secretions, though the constitutive secretion was shown to release VWF from basolateral position of endothelia cell membrane or to accumulate the VWF in the subendothelial matrix of endothelia cells which may promote platelets adhesion upon vascular injury [48-50].

Earlier study by Jaulin et al. had suggested that Kif5b takes part in p75 apical transportation in polarized MDCK cells [48]. However, Cui et al. reported that Kif5b may be involved in basolateral transportation of $\mathrm{Na}^{+}-\mathrm{K}^{+}$ATPase-containing vesicles in epithelial cells [51]. Taken together, this indicates that Kif5b may participate in both apical and basolateral transportations. Furthermore, a few papers have reported that kinesin(s) collaborate with dynein along microtubules after VWF biosynthesis in TGN, while the type of kinesin(s) involved in this process is not defined $[1,13]$. Considering our finding that knockdown of Kif5b results in increasing constitutive secretion of VWF both in vitro and in vivo, we hypothesize Kif5b might participate and play a major role in the basal secretion and regulated secretion of VWF, while there could be other unidentified kinesins involved in the constitutive secretion pathways.

In summary, normal hemostasis requires VWF to be processed into a desired multimeric form and to be secreted at a decent level and in a timely manner [44]. It is also important to keep an appropriate ratio between apical and basolateral VWF secretions. Kif5b seems to be critical for the maintenance of normal processing and secretion of VWF. Taken together, reduction of Kif5b apparently increases constitutive VWF secretion, impedes VWF maturation process, and at the same time prolongs bleeding time in mice which could be supported by the less high molecular weight VWF multimers observed in $\mathrm{Kif}^{\mathrm{b}} \mathrm{b}^{+/-}$mice. To our knowledge, it's the first report to elucidate the Kif5b modulates the processing and secretion of VWF in vitro and in vivo. Such findings raise more interesting questions that should be addressed with further wet lab studies.

\section{Author Contributions}

YSL and JDH designed this study. YSL, YZ and JDH wrote the manuscript. YSL performed experiments. YSL, YZ, KZ, XDL, EH, and JDH interpreted the findings, revised and proof reading the manuscript. $\mathrm{YZ}$ is the principal investigator who supervised this study.

\section{Acknowledgements}

We sincerely thank Dr. MF Liu for sharing the shRNA plasmid for Kif5b knockdown and scramble shRNA plasmid. We sincerely thank Dr. ZG Duan for providing the Kif5b knockdown mice.

\section{Presentation}

All of the results of this study have not been presented in any form of poster or scientific conference.

\section{Funding}

This work was jointly supported by the Joint Foundation of Collaboration Project between Scientific and Technological Bureau of Guizhou Province and Colleges/Universities of Guizhou Province (LH[2016]7245) and the Young Scientists Cultivation Project of Education Bureau of Guizhou Province (gzwjkj2018-1-022) to YSL; the National Basic Research Program of China (973 Program, 2014CB745200) from the Ministry of Science and Technology of People's Republic of China, the Shenzhen Peacock Project (KQTD2015033117210153), the Shenzhen Science and Technology Innovation Committee Basic Science Research Grant (JCYJ20150629151046896), and the General Research Fund of the Research Grants Council of Hong Kong Special Administrative Region (HKU 17127015) to JDH; the National Science Foundation of Guangdong Province Grant (2015A030313745) and the Shenzhen Science and Technology Innovation Committee Basic Science Research Grants (JCYJ20140901003939019) to YZ.

\section{Reference}

1. Chamberlain A (2013) The secretion of von Willebrand factor from endothelial cells; an increasingly complicated story. J Thromb Haemost 11: 192-201.

2. Ruggeri ZM (2007) VonWillebrand factor: Looking back and looking forward Thromb Haemost 98: 55-62.

3. Michaux G, Abbitt KB, Collinson LM, Haberichter SL, Norman KE, et al. (2006) The physiological function of von Willebrand's factor depends on its tubular storage in endothelial Weibel-Palade bodies. Dev Cell 10: 223-232.

4. Mourik JAV, Wit TRD, Voorberg J (2002) Biogenesis and exocytosis of Weibel-Palade bodies. Histochem Cell Biol 117: 113-122.

5. Kanaji S, Fahs SA, Shi Q, Haberichter SL, Montgomery RR (2012) Contribution of platelet vs. endothelial VWF to platelet adhesion and hemostasis. J Thromb Haemost 10: 1646-1652.

6. Lopes DSM, Cutler DF (2016) Von Willebrand Factor multimerization and the polarity of secretory pathways in endothelial cells. Blood 128: 277-285.

7. De Ceunynck K, De Meyer SF, Vanhoorelbeke K (2013) Unwinding the von Willebrand factor strings puzzle. Blood 121: 270-277.

8. Tsai HM, Nagel RL, Hatcher VB, Seaton AC, Sussman II (1991) The high molecular weight form of endothelial cell von Willebrand factor is released by the regulated pathway. $\mathrm{Br} \mathrm{J}$ Haematol 79: 239-245.

9. Luo GP, Ni B, Yang X, Wu YZ (2012) von Willebrand factor: more than a regulator of hemostasis and thrombosis. Acta Haematol 128: 158-169.

10. Trossaërt M, Ternisien C, Lefrancois A, Llopis L, Goudemand J, et al. (2011) Evaluation of an automated von Willebrand factor activity assay in von Willebrand disease. Clin Appl Thromb Hemost 17: E25-29.

11. Schwameis M, Schörgenhofer C, Assinger A, Steiner MM, Jilma B (2015) VWF excess and ADAMTS13 deficiency: a unifying pathomechanism linking inflammation to thrombosis in DIC, malaria, and TTP. Thromb Haemost 113 708-718.

12. Hopkins SC, Vale RD, Kuntz ID (2000) Inhibitors of kinesin activity from structure-based computer screening. Biochemistry 39: 2805-2814.

13. Manneville JB, Etienne MSP, Carter T, Ogden D, Ferenczi M (2003) Interaction of the actin cytoskeleton with microtubules regulates secretory organelle movement near the plasma membrane in human endothelial cells. J Cell Sci 116: 3927-3938.

14. Hirokawa N, Pfister KK, Yorifuji H, Wagner MC, Brady ST, et al. (1989) Submolecular domains of bovine brain kinesin identified by electron microscopy and monoclonal antibody decoration. Cell 56: 867-878.

15. Hirokawa N, Noda Y, Tanaka Y, Niwa S (2009) Kinesin superfamily motor proteins and intracellular transport. Nat Rev Mol Cell Biol 10: 682-696.

16. Kanai Y, Okada Y, Tanaka Y, Harada A, Terada S, et al. (2000) KIF5C, a novel neuronal kinesin enriched in motor neurons. J Neurosci 20: 6374-6384.

17. Tanaka Y, Kanai Y, Okada Y, Nonaka S, Takeda S, et al. (1998) Targeted disruption of mouse conventional kinesin heavy chain kif5B, results in abnormal perinuclear clustering of mitochondria. Cell 93: 1147-1158.

18. Xia CH, Roberts EA, Her LS, Liu X, Williams DS, et al. (2003) Abnormal neurofilament transport caused by targeted disruption of neuronal kinesin heavy chain KIF5A. J Cell Biol 161: 55-66.

19. Ma H, Cai Q, Lu W, Sheng ZH, Mochida S (2009) KIF5B motor adaptor syntabulin maintains synaptic transmission in sympathetic neurons. J Neurosc 29: $13019-13029$ 
Citation: Yu-Si Luo, Yue Zhuo, Ke Zhang, Xingde Liu, Erhard Hofer, et al. (2018) Kif5b Modulates Processing and Secretion of von Willebrand Factor in Endothelial Cells and Mice. Cardiovasc Pharm Open Access 7: 249. doi: 10.4172/2329-6607.1000249

Page 8 of 8

20. Chen $\mathrm{Y}$, Sheng ZH (2013) Kinesin-1-syntaphilin coupling mediates activitydependent regulation of axonal mitochondrial transport. J Cell Biol 202: 351-364

21. Cho KI, Cai Y, Yi H, Yeh A, Aslanukov A, et al. (2007)Association of the kinesin-binding domain of RanBP2 to KIF5B and KIF5C determines mitochondria localization and function. Traffic 8: 1722-1735.

22. Chen X, Kojima SI, Borisy GG, Green KJ (2003) p120 catenin associates with kinesin and facilitates the transport of cadherin-catenin complexes to intercellular junctions. J Cell Biol 163: 547-557.

23. Grigoriev I, Splinter D, Keijzer N, Wulf PS, Demmers J, et al. (2007) Rab6 regulates transport and targeting of exocytotic carriers. Dev Cell 13: 305-314.

24. Cui J, Wang Z, Cheng Q, Lin R, Zhang XM, et al. (2011) Targeted inactivation of Kinesin-1 in pancreatic $\beta$-cells in vivo leads to insulin secretory deficiency. Diabetes 60: 320-330.

25. Wang Z, Cui J, Wong WM, Li X, Xue W, et al. (2013) Kif5b controls the localization of myofibril components for their assembly and linkage to the myotendinous junctions. Development 140: 617-626.

26. Sinha S, Wagner D (1987) Intact microtubules are necessary for complete processing, storage and regulated secretion of von Willebrand factor by endothelial cells. Eur J Cell Biol 43: 377-383.

27. Vischer UM, Barth H, Wollheim CB (2000) Regulated von Willebrand factor secretion is associated with agonist-specific patterns of cytoskeletal remodeling in cultured endothelial cells. Arter Thromb Vasc Biol 20: 883-891.

28. Zhang X (2004) Generation of mouse models to study intracellular transportation in Purkinje cells and melanocytes. Curr Opin Neurobiol 14: 259-263.

29. Thomas P, Smart TG (2005) HEK293 cell line: A vehicle for the expression of recombinant proteins. J Pharmacol Toxicol Methods 51: 187-200.

30. Ventura A, Meissner A, Dillon CP, McManus M, Sharp PA, et al. (2004) Crelox-regulated conditional RNA interference from transgenes. Proc Natl Acad Sci USA 101: 10380-10385.

31. Zhao BQ, Chauhan AK, Canault M, Patten IS, Yang JJ, et al. (2009) von Willebrand factor-cleaving protease ADAMTS13 reduces ischemic brain injury in experimental stroke. Blood 114: 3329-3334

32. Torisu T, Torisu K, Lee IH, Liu J, Malide D, et al. (2013) Autophagy regulates endothelial cell processing, maturation and secretion of von Willebrand factor. Nat Med 19: 1281-1287.

33. Zenner HL, Collinson LM, Michaux G, Cutler DF (2007) High-pressure freezing provides insights into Weibel-Palade body biogenesis. J Cell Sci 120: 2117 2125.

34. Ott HW, Griesmacher A, Schnapka-Koepf M, Golderer G, Sieberer A, et al (2010) Analysis of von Willebrand factor multimers by simultaneous high- and low-resolution vertical SDS-agarose gel electrophoresis and Cy5-labeled antibody high-sensitivity fluorescence detection. Am J Clin Pathol 133: 322-330.

35. Zhao H, Zhou J, Zhang K, Chu H, Liu D, et al. (2015) A novel peptide with potent and broad-spectrum antiviral activities against multiple respiratory viruses. Sci Rep 6: 1.
36. Erent M, Meli A, Moisoi N, Babich V, Hannah MJ, et al. (2007) Rate extent and concentration dependence of histamine-evoked Weibel-Palade body exocytosis determined from individual fusion events in human endothelial cells. J Physiol 583: 195-212.

37. Vischer UM, Barth $H$, Wollheim CB (2000) Regulated von Willebrand factor secretion is associated with agonist-specific patterns of cytoskeletal remodeling in cultured endothelial cells. Arter Thromb Vasc Biol 20: 883-891.

38. Hirokawa N, NodaY (2008) Intracellular transport and kinesin superfamily proteins, KIFs: structure, function, and dynamics. Physiol Rev 88: 1089-1118.

39. Gindhart JG (2006) Towards an understanding of kinesin-1 dependent transport pathways through the study of protein-protein interactions. Brief Funct Genomic Proteomic 5: 74-86.

40. Seiler S, Kirchner J, Horn C, Kallipolitou A, Woehlke G, et al. (2000) Cargo binding and regulatory sites in the tail of fungal conventional kinesin. Nat Cell Biol 2: 333-338.

41. Diefenbach RJ, Mackay JP, Armati PJ, Cunningham AL (1998) The C-termina region of the stalk domain of ubiquitous human kinesin heavy chain contains the binding site for kinesin light chain. Biochemistry 37: 16663-16670.

42. Kanai Y, Dohmae N, Hirokawa N (2004) Kinesin transports RNA: isolation and characterization of an RNA-transporting granule. Neuron 43: 513-525.

43. Bi GQ, Morris RL, Liao G, Alderton JM, Scholey JM, et al. (1997) Kinesinand myosin-driven steps of vesicle recruitment for $\mathrm{Ca}^{2+}$-regulated exocytosis. J Cell Biol 138: 999-1008.

44. Lenting PJ, Christophe OD, Denis CV (2015) von Willebrand facto biosynthesis, secretion, and clearance: connecting the far ends. Blood 125 : 2019-2028.

45. Roldán V, Marín $F$, Muiña $B$, Torregrosa JM, Hernández-Romero $D$, et al. (2011) Plasma von Willebrand factor levels are an independent risk factor for adverse events including mortality and major bleeding in anticoagulated atria fibrillation patients. J Am Coll Cardiol 57: 2496-2504.

46. Valentijn KM, Valentijn JA Jansen KA Koster AJ (2008) A new look at Weibel-Palade body structure in endothelial cells using electron tomography. J Struct Biol 161: 447-458.

47. Stockschlaeder M, Schneppenheim R, Budde U (2014) Update on von Willebrand factor multimers: focus on high-molecular-weight multimers and their role in hemostasis. Blood Coagul Fibrinolysis 25: 206-216.

48. Jaulin F, Xue X, Rodriguezboulan E, Kreitzer G (2007) Polarization-dependent selective transport to the apical membrane by KIF5B in MDCK cells. Dev Cell 13: $511-522$.

49. Zheng Y, Chen J, López JA (2015) Flow-driven assembly of VWF fibres and webs in in vitro microvessels. Nat Commun 6.

50. Bierings R, Voorberg J (2016) Up or out: polarity of VWF release. Blood 128 : 154-155.

51. Cui J, Li X, Duan Z, Xue W, Wang Z, et al. (2015) Analysis of Kif5b expression during mouse kidney development. Plos One 10: e0126002. 